\title{
Les bulles de travail : Comment les entreprises peuvent-elles rouvrir en réduisant le risque d'éclosions de la COVID-19?
}

\author{
Jeffrey Shaw MD MSc, Troy Day PhD, Nadia Malik BIng MBA, Nancy Barber BA, Hayley Wickenheiser MSc, \\ David N. Fisman MD MSP, Isaac Bogoch MD MSc, John S. Brownstein PhD, Tyler Williamson PhD
}

Citation : CMAJ 2020 November 2;192:E1362-6. doi : 10.1503/cmaj.201582-f; diffusion hâtive le 30 septembre 2020

Voir la version anglaise de l'article ici : www.cmaj.ca/lookup/doi/10.1503/cmaj.201582

$\mathbf{E}$

$\mathrm{n}$ raison de la pandémie de la maladie à coronavirus 2019

(COVID-19), les gouvernements partout au monde ont dû adopter de strictes mesures d'éloignement physique pour réduire la propagation du virus afin de protéger la santé publique et de préserver la capacité du système de santé. Les mesures de prévention dans beaucoup de pays, notamment au Canada, ont inclus la fermeture temporaire des entreprises non essentielles, ce qui a entraîné des perspectives sombres pour l'emploi et l'économie ${ }^{1}$. Les entreprises essentielles qui ont pu garder leurs portes ouvertes ont, dans bien des cas, contribué à propager la pandémie. Les usines dont les employés travaillent très près les uns des autres ont été particulièrement touchées; non seulement ont-elles mis en péril la santé et la sécurité des travailleurs, mais elles ont nui aux chaînes d'approvisionnement et aux entreprises en $\mathrm{aval}^{2}$. Il y a un compromis à faire entre, d'une part, la nécessité de protéger les populations des effets directs de l'infection par le coronavirus du syndrome respiratoire aigu sévère 2 (SRAS-CoV-2) et, d'autre part, les effets indirects mais bien réels sur la santé des restrictions imposées aux activités commerciales et économiques ${ }^{3}$.

À mesure que les provinces et territoires du Canada assouplissent les mesures de santé publique et permettent aux entreprises non essentielles de rouvrir, 2 défis de taille se posent : les entreprises doivent assurer la sécurité des employés et continuer de rentabiliser leurs activités, en tenant compte des restrictions imposées par les autorités de santé publique. C'est pourquoi il est essentiel que toute stratégie de retour au travail comprenne des mesures visant à prévenir l'infection de travailleurs par le SRAS-CoV-2 et un plan d'intervention rapide dans les cas d'infections possibles afin d'endiguer la propagation, si le virus parvenait à s'infiltrer. Nous analysons des stratégies qui ont été mises de l'avant afin de protéger les employés dans le cadre de l'assouplissement des mesures de santé publique et de limiter le risque d'éclosion à l'échelle de toute une entreprise. Nous abordons également une approche qui consiste à regrouper les employés en cohortes, qui sont organisées en fonction de

\section{POINTS CLÉS}

- Il y a un compromis à faire entre la nécessité de protéger les populations des effets directs de l'infection par le coronavirus du syndrome respiratoire aigu sévère 2 (SRAS-CoV-2), d'une part, et les effets indirects mais bien réels sur la santé des restrictions imposées aux activités commerciales et économiques, d'autre part.

- Certaines entreprises, y compris des services essentiels, ont été touchées par de vastes éclosions qui ont eu de graves conséquences sur les travailleurs et la communauté avoisinante.

- Les employeurs ont la responsabilité d'offrir un milieu de travail sécuritaire à leurs employés.

- La création de cohortes au sein d'une entreprise peut efficacement réduire le risque d'éclosions à l'échelle de toute l'entreprise; cette approche a été appliquée avec succès par une grande société canadienne.

l'horaire, de l'espace ou des 2 éléments, ainsi que des règles connexes qui visent à prévenir la propagation du SRAS-CoV-2 et à contenir rapidement les éclosions dans les milieux de travail. Enfin, nous donnons un exemple de la façon dont cette approche a été appliquée par une entreprise canadienne, Bombardier, qui gère 7 usines dans 2 pays et dans 4 provinces et états, et qui emploie 25000 personnes (10 000 au Canada).

\section{Propagation du SRAS-CoV-2 dans les milieux de travail : Quelle est l'ampleur du problème?}

La propagation du SRAS-CoV-2 a été en grande partie attribuable aux contacts en milieu de travail ${ }^{4}$. Par exemple, le 11 mai 2020 en Alberta, il y avait 6183 cas au total, dont 5539 qui n'étaient pas associés à des centres de soins de longue durée ${ }^{5}$. Des reportages ${ }^{6}$ indiquent que, de ce nombre, 1783 cas (32,1\% des cas non institutionnels) étaient liés à une exposition au virus en milieu de travail. Lorsqu'on inclut la transmission communautaire élargie qui 
est associée à ces éclosions, la proportion s'élève à près de $50 \%$ de tous les cas. L'usine de transformation de viandes de Cargill, dans la région de Calgary, a été qualifiée de la pire éclosion au Canada, car elle a été directement responsable de 941 cas et indirectement responsable de 600 cas de plus dans la communauté avoisinante. Des observations similaires ont été faites dans 6 pays d'Asie, où on estime que $15 \%$ d'un total de 690 cas de transmission locale étaient liés au travail'. L'éclosion dans l'« immeuble de bureaux X » en Corée du Sud a nécessité l'isolement de plus de 1200 travailleurs d'une tour de bureaux après que $44 \%$ des employés d'un centre d'appel dans la tour ont contracté le virus ${ }^{8}$. Ces éclosions soulèvent d'importantes préoccupations en matière de santé et perturbent les activités des entreprises. Dans chacun de ces exemples, l'absence d'éloignement physique entre les employés et l'incapacité de rapidement contenir l'éclosion sont des facteurs qui ont grandement contribué à la transmission. Même si les employeurs ont la responsabilité d'offrir un milieu de travail sécuritaire à leur personnel, l'application des mesures d'éloignement physique et d'endiguement rapides peut être problématique, voire impossible, pour beaucoup d'organisations dans bon nombre de secteurs d'activités.

Bombardier Aéronautique menait des activités dans 2 des villes les plus touchées au pays, soit Montréal et Toronto. Même si elle a suivi de près les recommandations en matière de santé publique, l'entreprise a signalé son premier cas de COVID-19 en mars à l'une de ses usines mondiales de fabrication aéronautique, où travaillent 900 employés, ce qui a obligé l'entreprise à cesser immédiatement ses activités. L'usine a été fermée pendant 3 jours consécutifs pour que les installations soient désinfectées en profondeur et pour qu'il soit possible de communiquer avec les employés ayant pu être infectés; ceux qui avaient eu des contacts étroits ont dû s'isoler pendant 2 semaines. Toute personne qui avait été en contact avec des cas confirmés et qui s'était rendue dans une autre installation a également été retracée, et les mesures appropriées ont été prises pour désinfecter des zones précises ou exiger que des personnes se placent en isolement. Étant donné que le secteur de la fabrication aéronautique est associé à de longs délais de production et qu'il nécessite d'importants investissements pour accumuler des stocks, il est essentiel de réduire la durée du cycle de fabrication. Cet objectif est généralement atteint en faisant travailler de nombreux employés en même temps par quart de travail, ce qui complique l'éloignement physique. En général, des centaines d'employés entrent dans un immeuble et en ressortent dans un court laps de temps; ils doivent tous utiliser les ascenseurs, les vestiaires communs, les toilettes et les services de cafétéria. Dans le secteur de la fabrication aéronautique, en particulier, les employés doivent travailler près les uns des autres lorsqu'ils construisent l'intérieur d'un aéronef, par exemple dans l'espace étroit et confiné d'un fuselage, dans des conditions où la distance de 2 mètres ne peut pas toujours être respectée et où la circulation de l'air n'est pas optimale. Ces conditions sont favorables à la propagation du SRAS-CoV-2.

La pandémie a fait ressortir l'existence de conditions de travail semblables dans plusieurs autres secteurs, y compris le secteur manufacturier et celui de la transformation alimentaire, et bon nombre d'entre eux ont connu des éclosions inquiétantes. Cependant, la nécessité pour les employés de travailler en contact étroit pendant des périodes prolongées n'est pas un problème qui existe seulement dans les grandes entreprises. En effet, les employés de beaucoup de petites entreprises doivent aussi travailler dans des conditions semblables.

\section{Que peut-on faire pour limiter la propagation du SRAS-CoV-2 dans les milieux de travail?}

Si des cohortes sont conçues selon les principes de la redondance et des unités de travail fonctionnelles, il est possible d'optimiser la sécurité des employés et la résilience des entreprises.

\section{L'éloignement physique au moyen de bulles de travail}

Il est largement accepté que les mesures d'éloignement physique ont considérablement réduit la transmission du SRAS-CoV- $2^{9-11}$. Beaucoup de gens ont respecté cet éloignement physique en travaillant à domicile, lorsque c'était possible. Cependant, dans le cas des travailleurs qui doivent être présents sur le lieu de travail, il n'est pas toujours possible de maintenir un éloignement physique adéquat, car des groupes d'employés peuvent partager des espaces communs ou se trouver près les uns des autres. Pour équilibrer le besoin d'éloignement physique et la nécessité de se trouver sur le lieu de travail, un employeur peut notamment organiser son personnel en cohortes, pour que les employés interagissent dans leur groupe sans avoir d'interactions avec d'autres cohortes. Cette approche, qui consiste à créer ce qu'on pourrait appeler des "bulles de travail ", ressemble à celle des "bulles sociales». Selon les contraintes logistiques d'une entreprise, ces bulles de travail peuvent être créées de plusieurs façons, en ayant recours à une séparation physique dans un espace de travail ou à une séparation temporelle au moyen d'un horaire de travail rotatif, ou les 2.

Ce type d'organisation de la main-d'œuvre procure plusieurs avantages, tant sur le plan de la santé publique que sur le plan de la sécurité et du bien-être des employés et des employeurs (encadré 1). La figure 1 présente le taux de reproduction de base en fonction de l'efficacité des mesures d'éloignement physique selon 3 différents scénarios. La ligne noire représente l'arrêt des activités qui a eu lieu en avril et en mai, lorsque seuls les services essentiels étaient en activité. La ligne pleine verte représente le scénario dans lequel tous les employés retournent au travail 3 jours par semaine, sans aucune autre stratégie d'atténuation. Quant à la ligne hachurée bleue, elle représente le scénario dans lequel tous les employés retournent au travail 3 jours par semaine, mais en 2 bulles de travail séparées dans le temps qui travaillent en alternance. Il est évident que le fait d'organiser 2 bulles de travail travaillant selon des horaires différents peut réduire la transmission interpersonnelle dans la communauté. De plus, si les cohortes sont complètement séparées, il est possible de réduire en même temps le risque d'une fermeture complète de l'entreprise si un employé reçoit un résultat positif à un test de dépistage. 
Santé publique

\section{Avantages}

- Ce modèle peut réduire le taux de reproduction de la maladie (figure 1).

- Il peut accroître l'efficience et l'efficacité des systèmes de recherche de contacts en offrant un outil pour assurer le retrait immédiat de personnes à risque de leur milieu de travail.

- Il s'agit d'une approche préventive pour la recherche de contacts entre les employés qui réduit la propagation non seulement au sein d'une entreprise, mais également dans la communauté avoisinante en offrant un outil pour assurer l'isolement rapide des contacts.

Défis

- Ce modèle est difficile à appliquer.

\section{Personnel Avantages}

- Ce modèle offre une autre façon de protéger les employés contre la possibilité de contracter la COVID-19 au travail.

- Il peut améliorer la confiance des employés envers la sécurité dans le milieu de travail.

Défis

- Ce modèle peut nuire à l'horaire de travail.

- Il se peut que les employés ne puissent pas socialiser avec les personnes en dehors de leur bulle de travail.

- La productivité peut s'en trouver diminuée.

\section{Employeurs Avantages}

- Si les bulles de travail au sein d'une entreprise sont modulaires et redondantes, il est possible de retirer certains bulles sans interrompre le reste des activités de l'entreprise.

- Ce modèle permet aux employeurs de prendre des mesures préventives actives pour assurer la sécurité de leurs employés.

Défis

- Ce modèle accroît la complexité administrative liée à l'établissement des horaires du personnel.

- Il peut être difficile de s'assurer que les bulles de travail restent séparées l'une de l'autre.

$\mathbf{R}_{0}$

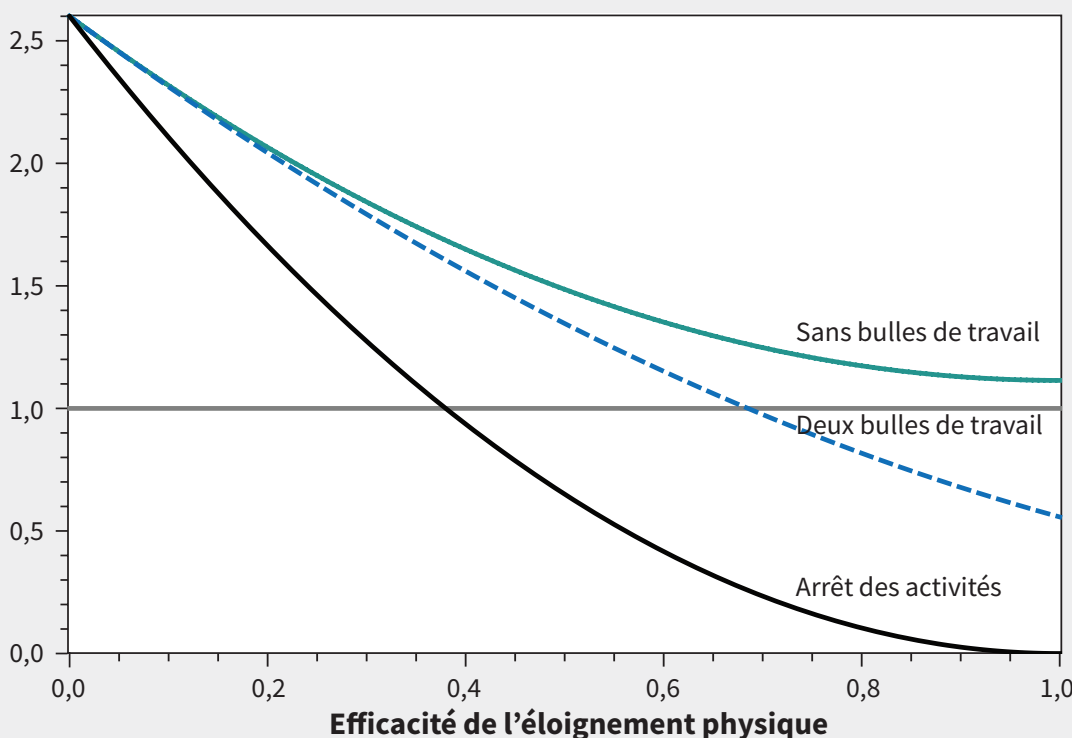

Figure 1 : Taux de reproduction de base en fonction de l'efficacité de l'éloignement physique selon 3 scénarios : la période actuelle d'arrêt des activités, durant laquelle seuls les services essentiels sont en activité (ligne noire), le scénario selon lequel tous les employés retournent au travail 3 jours par semaine sans aucune autre stratégie d'atténuation (ligne pleine verte), et le scénario selon lequel tous les employés retournent au travail 3 jours par semaine, mais en 2 bulles de travail séparées dans le temps qui travaillent en alternance (ligne hachurée bleue). Voir la méthodologie à l'annexe 1 (accessible en anglais ici : www.cmaj.ca/lookup/doi/10.1503/cmaj.201582/tab-related-content).

Chez Bombardier, les employés de bureau (p. ex., ceux des services de génie, des finances, de la gestion et des programmes) qui pouvaient travailler à domicile et obtenir tous les résultats attendus pour leur poste ont été tenus de le faire. Grâce à cette stratégie, seuls les employés qui participaient à la construction et à la livraison d'aéronefs devaient se trouver sur place. Pour ces travailleurs, la méthode de fabrication de l'entreprise pour les employés syndiqués reposait déjà sur des cohortes (c'est-à-dire 
des quarts de travail ayant différents horaires), et Bombardier a mis en place une autre forme de bulles de travail physiques et temporelles qui se fondait sur les principes suivants ${ }^{1}$ :

- Les bulles de travail fonctionnelles doivent compter le plus petit nombre de personnes nécessaires pour accomplir le travail ${ }^{2}$.

- Les bulles de travail doivent être conçues pour permettre aux activités de l'entreprise de se poursuivre même si l'une des bulles doit être retirée de l'effectif ${ }^{3}$.

- Les bulles de travail doivent être strictement séparées dans le temps ou l'espace, ou les 2, afin d'éliminer le risque de transmission entre elles. Cette séparation peut être obtenue par la rotation des jours de travail ou par l'éloignement physique, en assurant la décontamination méticuleuse des espaces communs après leur utilisation par une bulle de travail ${ }^{4}$.

- Un délai d'attente de 5 jours doit idéalement être prévu avant de faire passer des personnes d'une bulle de travail à une autre, pour tenir compte du temps d'incubation du virus.

Bombardier a également favorisé l'éloignement physique en échelonnant les heures d'arrivée et de départ des employés sur le lieu de travail, ce qui a permis d'éliminer les rencontres entre les différentes cohortes qui arrivent dans l'installation ou en repartent, et a aussi échelonné leur accès aux aires communes comme les vestiaires et les cafétérias. Le renforcement et des changements à la stratégie d'établissement de cohortes ont mené à des discussions fructueuses avec les syndicats, et les horaires des quarts ont été modifiés pour limiter la population présente dans l'installation durant un quart donné. De même, Bombardier a décidé que les cohortes ne se déplaceraient pas librement d'un site à l'autre, mais demeureraient à un seul endroit, pour réduire encore plus les interactions entre les employés. Plus précisément, Bombardier a augmenté les heures de travail de ses cohortes qui font des quarts de travail de semaine (jour, soir, nuit), les faisant passer de quarts de 8 heures à des quarts de 10 heures. Les employés de fin de semaine ont conservé leurs quarts de 12 heures, car ils constituaient déjà un plus petit groupe d'employés et une cohorte établie. Les changements apportés aux cohortes temporelles ont permis à l'entreprise de bien gérer le nombre d'employés se trouvant dans les aires communes, compte tenu du nombre réduit d'employés sur place, et de désinfecter ces aires plusieurs fois par jour et entre les quarts de travail. Ces modifications ont aussi permis de garantir que les activités de l'entreprise pourront continuer si un employé recevait un résultat positif à un test de dépistage du SRAS-CoV-2, car l'exposition serait limitée à sa propre cohorte.

Pour mieux protéger les employés et réduire le potentiel de transmission, un modèle préexistant de " cohorte à l'intérieur d'une cohorte » a également été mis en place. Maintenant, les employés se consacrent à travailler sur un aéronef particulier, sans avoir accès à un autre aéronef ou à une différente cohorte d'employés. Même si cette mesure était essentielle pour la sécurité, elle continue de poser des défis pour l'organisation, car les employés ont des compétences et des expertises différentes qui peuvent être requises dans plusieurs cohortes en même temps.

\section{Dépistage et isolement rapides des cas symptomatiques}

Un autre pilier de l'approche de santé publique de lutte contre la COVID-19 a été le dépistage précoce et l'isolement des cas. Cette stratégie a permis de limiter la transmission de la maladie en Australie, en Corée du Sud et à Hong Kong grâce à une recherche de contacts approfondie et rapide ${ }^{12-14}$. Cependant, la réussite globale de cette stratégie est directement liée à la vitesse à laquelle les personnes ayant des symptômes se mettent en isolement, à la disponibilité des tests et à la capacité du système à effectuer des recherches de contacts ${ }^{12,14}$.

Lorsqu'il est combiné à l'utilisation de bulles de travail, le dépistage quotidien des symptômes est largement considéré comme une autre façon de réduire la probabilité d'une éclosion au sein d'un groupe de travailleurs ${ }^{15}$. Par exemple, l'organisme Alberta Health Services a ordonné à tous les travailleurs de la santé de remplir un outil électronique d'autodépistage avant d'entrer dans leur lieu de travail ${ }^{15}$. Les employés ayant des symptômes s'apparentant à la COVID-19 (fièvre, toux, etc.) doivent rester à la maison, ne pas se présenter au travail et, si les autorités locales de la santé publique le demandent, aller subir un test. Étant donné que les tests sont gérés par les gouvernements provinciaux au Canada, les stratégies de détection rapide sont largement en dehors du ressort des employeurs. Cependant, les gouvernements doivent faire tous les efforts possibles pour garantir des tests de dépistage rapides aux personnes symptomatiques et aux personnes ayant eu un contact possible avec une personne atteinte de la COVID-19. Des tests sont offerts aux personnes asymptomatiques dans certaines provinces, ce qui permet une détection encore plus précoce de la COVID-19, mais ils ne sont pas généralement recommandés ${ }^{16}$.

Si les bulles de travail sont bien gérées, un employé qui reçoit un résultat positif à un test de dépistage du SRAS-CoV-2 peut aviser son employeur, pour que les membres de sa bulle de travail reçoivent rapidement l'instruction de s'isoler. Cette stratégie d'isolement rapide est avantageuse à la fois pour la santé publique, en permettant un repérage plus rapide des contacts en milieu de travail, et pour l'employeur, en limitant l'exposition aux personnes d'une même bulle de travail, ce qui peut permettre de contenir l'éclosion.

\section{Conclusion}

À mesure que nous commençons à assouplir les mesures de santé publique qui ont été mises en place pour ralentir la propagation de la COVID-19 au Canada, il est essentiel de songer à la façon de limiter le risque de contracter la maladie dans le lieu de travail. L'adoption d'une stratégie axée sur des bulles de travail, qui permet de séparer les employés dans l'espace ou dans le temps (ou les 2) au moyen d'horaires de travail modifiés, réduira le risque de transmission de la maladie à l'échelle d'une entreprise ainsi que le risque d'un arrêt complet des activités. Il existe des obstacles pratiques à la mise en place de ces stratégies, comme l'expérience de Bombardier l'a montré, mais avec une planification rigoureuse et une aide technique, il est possible de réduire les risques associés à un retour dans le milieu de travail. 


\section{Références}

1. Business Outlook Survey - Spring 2020. Ottawa: Bank of Canada. 2020; 2020 Apr. 6. Accessible ici : www.bankofcanada.ca/2020/04/business-outlook -survey-spring-2020/ (consulté le 10 septembre 2020).

2. Ivanov D. The impacts of epidemic outbreaks on global supply chains: a simulation-based analysis on the coronavirus outbreak (COVID-19/SARS-CoV-2) case. Transp Res E Logist Trans Rev Avril 2020;136 101922. doi : 10.1016/j.tre.2020.10192.

3. Glover A, Heathcote J, Krueger D, et al. Health versus wealth: on the distributional effects of controlling a pandemic. NBER Working Paper Series No. 27046. Cambridge (MA) : National Bureau of Economic Research; 2020.

4. Koh D. Occupational risk for COVID-19 infection [éditorial]. Occup Med (Lond) 2020;70:3-5.

5. Chief medical officer of health COVID-19 update - May 11, 2020: Alberta's chief medical officer of health provides an update on COVID-19 and the ongoing work to protect public health. Government of Alberta; 2020 May 11. Accessible ici : www.alberta.ca/release.cfm?xID=713293A50B3C8-ED31-6A0E -B72A0FE682E3F1EC (consulté le 10 septembre 2020).

6. What you need to know about COVID-19 in Alberta on Saturday, May 16. CBC (Calgary); 16 mai 2020. Accessible ici : www.cbc.ca/news/canada/calgary/what -you-need-to-know-alberta-may-16-1.5573241 (consulté le 10 septembre 2020).

7. Lan F-Y, Wei C-F, Hsu Y-T, et al. Work-related COVID-19 transmission in six Asian countries/areas: a follow-up study. PLoS One 2020;15:e023358.

8. Park S, Kim Y, Yi S, et al. Coronavirus disease outbreak in call center, South Korea. Emerg Infect Dis 2020;26:1666-70.

9. Colbourne T. COVID-19: extending or relaxing distancing control measures. Lancet 2020;5:e236-7.

10. Tuite AR, Fisman DN, Greer AL. Mathematical modelling of COVID-19 transmission and mitigation strategies in the population of Ontario, Canada. CMAJ 2020;192:E497-505.

11. Flaxman S, Mishra S, Gandy A, et al. Estimating the number of infections and the impact of nonpharmaceutical interventions on COVID-19 in 11 European countries. London (UK): Imperial College London; 2020 Mar. 30. doi : https:// doi.org/10.25561/77731.

12. Keeling MJ, Hollingsworth TD, Read JM. The efficacy of contact tracing for the containment of the 2019 novel coronavirus (COVID-19). medRxiv 2020 Feb. 17. doi : https://doi.org/10.1101/2020.02.14.20023036.

13. Bi Q, Wu Y, Mei S, et al. Epidemiology and transmission of COVID-19 in Shenzhen China: analysis of 391 cases and 1286 of their close contacts. medRxiv 2020 Mar. 27. doi : https://doi.org/10.1101/2020.03.03.20028423

14. Anderson RM, Heesterbeek $\mathrm{H}$, Klinkenberg D, et al. How will country-based mitigation measures influence the course of the COVID 19 epidemic? Lancet 2020;395:931-4.

15. AHS Scientific Advisory Group. Effectiveness of screening programs for reducing the spread of COVID-19 in healthcare settings. In : COVID-19 Scientific Advisory Group rapid evidence report. Alberta Health Services; 2020 June 12. Accessible ici : www. albertahealthservices.ca/assets/info/ppih/if-ppih-covid-19-sag-effectiveness-of -workplace-screening-programs-rapid-review.pdf (consulté le 10 septembre 2020).

16. Overview of testing for SARS-CoV-2 (COVID-19). Atlanta : Centers for Disease Control and Prevention; mise à jour le 18 septembre 2020. Accessible ici : www.cdc.gov/ coronavirus/2019-ncov/hcp/testing-overview.html (consulté le 10 septembre 2020).
Intérêts concurrents : Isaac Bogoch déclare qu'il a déjà été conseiller auprès de BlueDot, une entreprise à vocation sociale qui surveille la propagation de nouvelles maladies infectieuses. Jeffrey Shaw, Tyler Williamson et Hayley Wickenheiser déclarent qu'ils ont créé Pandemic Solutions, qui aide les entreprises à appliquer cette idée au sein de leur personnel; l'idée a été conçue et communiquée à des collègues et à des responsables des orientations politiques bien avant la création de l'entreprise. Aucun autre intérêt concurrent déclaré.

Cet article a été révisé par des pairs.

Affiliations : Service des soins intensifs (Shaw), Faculté de médecine Cumming, Université de Calgary, Calgary (Alberta); Département de statistique et de mathématiques (Day), Département de biologie, Université Queen's, Kingston (Ontario); Stratégie client et livraison des aéronefs (Malik), et Industrialisation opérationnelle, empreinte et planification centrale (Barber), Bombardier Aéronautique, Montréal (Québec); Faculté de médecine Cumming (Wickenheiser), Université de Calgary, Calgary (Alberta).; École de santé publique Dalla Lana (Fisman), Université de Toronto, Toronto (Ontario); divisions de la médecine interne générale et des maladies infectieuses (Bogoch), Réseau universitaire de santé, Toronto General Hospital, Toronto (Ontario); Département de pédiatrie (Brownstein), Faculté de médecine de Harvard, Boston Children's Hospital, Boston (Massachusetts); Département des sciences de la santé communautaire (Williamson), Faculté de médecine Cumming, Université de Calgary, Calgary (Alberta).

Collaborateurs : Tous les auteurs ont contribué à l'élaboration et à la conception des travaux ainsi qu'à l'acquisition, à l'analyse et à l'interprétation des données. Tous les auteurs ont participé à la rédaction du manuscrit, en ont révisé de façon critique le contenu intellectuel important, ont donné leur approbation finale pour la version destinée à être publiée, et assument l'entière responsabilité de tous les aspects du travail.

Propriété intellectuelle du contenu : Il s'agit d'un article en libre accès distribué conformément aux modalités de la licence Creative Commons Attribution (CC BY-NC-ND 4.0), qui permet l'utilisation, la diffusion et la reproduction dans tout médium à la condition que la publication originale soit adéquatement citée, que l'utilisation se fasse à des fins non commerciales (c.-à-d., recherche ou éducation) et qu'aucune modification ni adaptation n'y soit apportée. Voir : https://creativecommons.org/licenses/by-nc-nd/4.0/.

Correspondance : Jeffrey Shaw, jashaw@ucalgary.ca 